\title{
Image Encryption Based on the General Approach for Multiple Chaotic Systems
}

\author{
Qais H. Alsafasfeh, Aouda A. Arfoa
}

Electrical Engineering Department, Tafila Technical University, Tafila, Jordan.

Email: qsafasfeh@ttu.edu.jo, aouda@ttu.edu.jo

Received April 10 ${ }^{\text {th }}$ 2011; revised July $5^{\text {th }}, 2011$; accepted July $13^{\text {th }}, 2011$.

\begin{abstract}
In the recent years, researchers developed image encryption methods based on chaotic systems. This paper proposed new image encryption technique based on new chaotic system by adding two chaotic systems: the Lorenz chaotic system and the Rössler chaotic system. The main advantage of this technique is stronger security, as is shown in the encryption tests.
\end{abstract}

Keywords: Lorenz Equations, Rossler Systems, Image Encryption, Chaos

\section{Introduction}

Recent researches of nonlinear dynamical systems have been increasingly based on Chaos [1]. Image encryption schemes have been increasingly studied to meet the demand for real-time secure image transmission over private or public networks. Conventional image encryption algorithm such as data encryption standard (DES), is not suitable for image encryption because of the special storage characteristics of an image [2] and weakness of low-level efficiency when the image is large [3].The chaos-based encryption has suggested a new and efficient way to deal with the intractable problem of fast and highly secure, image encryption.

Chaotic systems have many important properties, such as the sensitive dependence on initial conditions and system parameters, the density of the set of all periodic points and topological transitivity, etc. Most properties are related to some requirements such as mixing and diffusion in the sense of cryptography [4]. Matthews proposed a chaotic encryption algorithm in 1989 [5], since then, increasing researches of image encryption technology are based on chaotic systems [4,6-12]. These methods have high-level efficiency but also weakness, such as small key space and weak security and complexity to overcome these drawbacks. This paper proposes a image encryption scheme based on combining two chaotic systems (Lorenz and Rosslere) and compares the results with the other techniques. The paper is organized as follows. Section 2 discusses the proposed scheme. Section 3 presents experimental results. Section 4 to Section 7 dis- cuss and examine the new scheme by applying statistical tests test also the security of the chaotic encryption is discussed. Conclusions are drawn in Section 8.

\subsection{Lorenz Chaotic System}

The Lorenz equations are a fairly simple model in which to study chaos.

$$
\left.\begin{array}{l}
\dot{x}=\sigma(y-x) \\
\dot{y}=r x-y-x z \\
\dot{z}=x y-\beta z
\end{array}\right\}
$$

The arbitrary parameters $\sigma, r$ and $b>0$ and for this example are $\sigma=10, r=28, b=8 / 3$. This system of differential equations is then solved numerically using Matlab's ode 45 Runge Kutta routine. The results are plotted in Figure 1 [12].

\subsection{Rossler Chaotic System}

A system of three differential equations has a simpler strange attractor than Lorenz's. That the Rossler system has only one quadratic nonlinear $x z$ numerical integration shows this system has a strange attractor for $a=b=$ 0.2, $c=5.7$ as shown in Figure 2 [12].

$$
\left.\begin{array}{l}
\dot{x}=-y-z \\
\dot{y}=x+a y \\
\dot{z}=b+z(x-c)
\end{array}\right\}
$$

\section{The Proposed Scheme}

First, most researchers used chaotic image encryption 

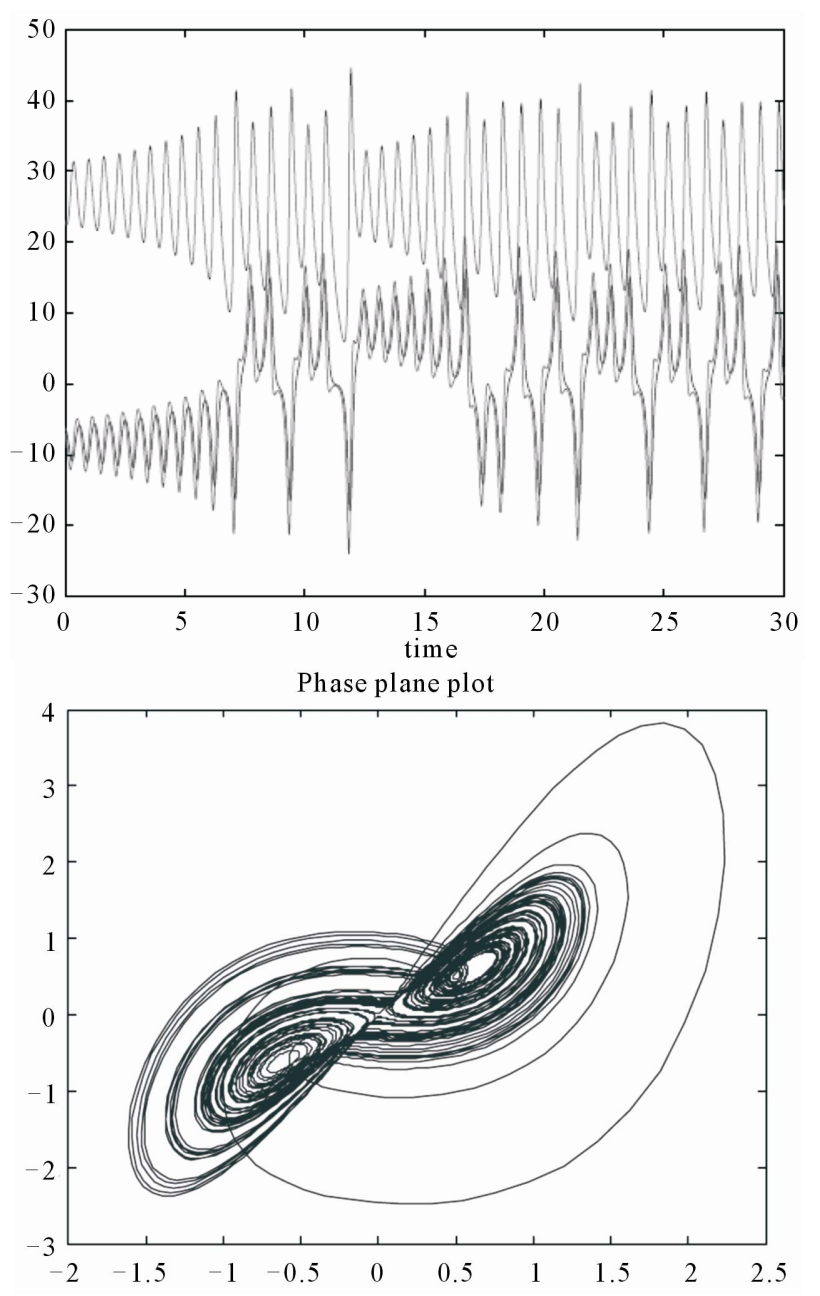

Figure 1. Chaotic system (time series and chaotic attractor for Lorenz system).

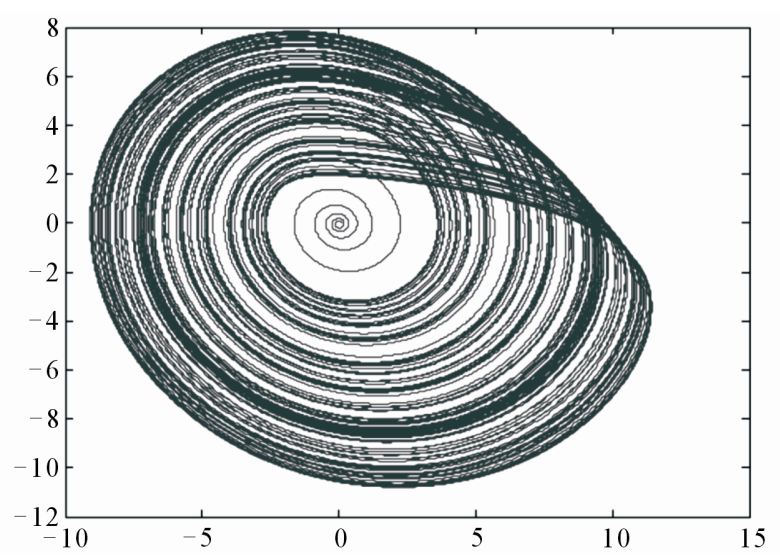

Figure 2. Chaotic system (Chaotic attractor for rossler system).

depending on only one chaotic system like Lorenz and Rossler systems. A chaotic was presented in [13], which is based on adding two chaotic systems (Lorenz and
Rossler), a new chaotic system was generated as shown in Equation (3).

$$
\left.\begin{array}{l}
\dot{x}=\sigma(y-x)-y-z \\
\dot{y}=r x-y-20 x z+x+a y \\
\dot{z}=5 x y-b z+s+x(z-c)
\end{array}\right\}
$$

Most of researchers agree on the following definition "chaos is aperiodic long term behavior in a deterministic system that exhibits dependence on initial condition" [12]. So to examine theses conditions we note in Figure $\mathbf{3}$ the new system has two attractors, thus satisfying the above definition.

The security of Lorenz and Rossler encryption methods depend on three parameters but in the proposed scheme in this paper the security level was increased to six parameters.

\section{Encryption Based Multiple Chaotic}

The goal of the step is to encrypt images by shuffling pixel values and then changing the grey scale values to create an encrypted image. The pixel values are rearranged using the XOR and then the grey scale values are changed using a multiple chaotic systems, and therefore after a set number of iterations (which depends on the size of the image), generated elements have been stored
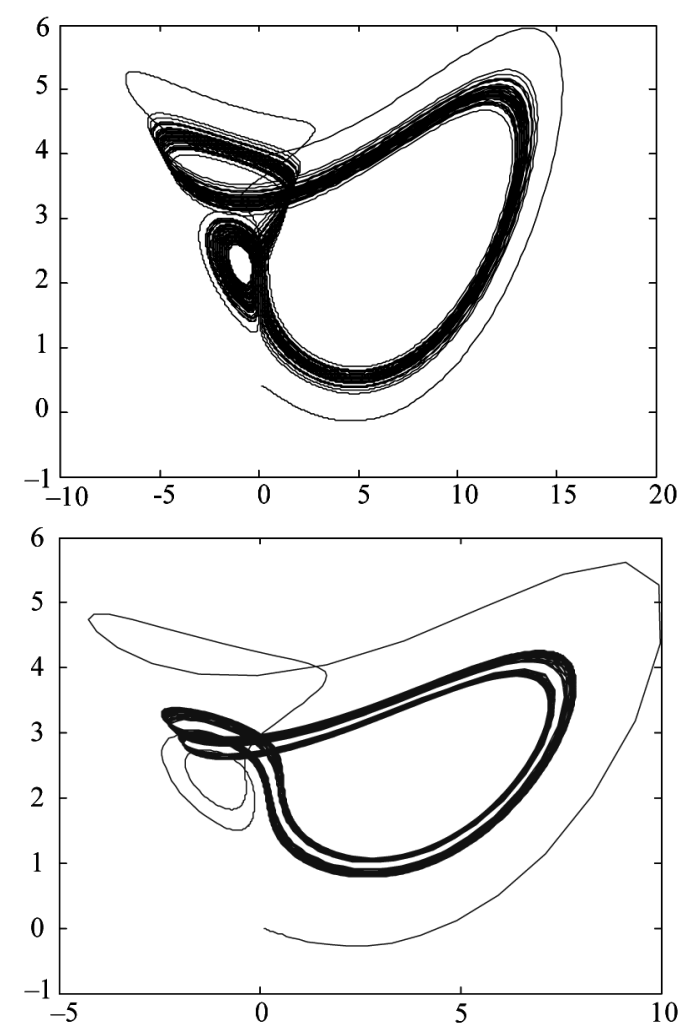

Figure 3. The phase plane for new system (Chaotic attractor projected onto $x y$-plane). 
within the chaotic matrix of size the same as the original image's size. As with the other algorithms that made use of the XOR operation to encrypt, decryption is a simple matter of recreating the matrix of chaotic elements and XOR with the decrypted image matrix and therefore after a set number of iterations the original image will return.

The actual procedure for encryption is shown in Figure 4.

Step 1. Set the key (initial condition and parameters $\left(\delta, \beta, r, a, b, c, y_{0}, x_{0}, z_{0}\right)$ in the acceptable intervals.

Step 2. Generate the first mask with the same size of image.

Step 3. Perform the XOR between the chaotic mask and original image.

Step 4. If the image is not encrypted generate the second mask with the same size of image.

Step 5. Perform the XOR between the chaotic mask and image in Step 3.

Step 6. If the image is not encrypted generate the third mask with the same size of image.

Step 7. Perform the XOR between the chaotic mask and image in Step 5.

Step 8. Pass the encrypted image

Step 9. Pass the encryption key.

Step 10. End.

\section{Experimental Results}

Simulation results and performance analysis of the proposed image encryption scheme are provided in this

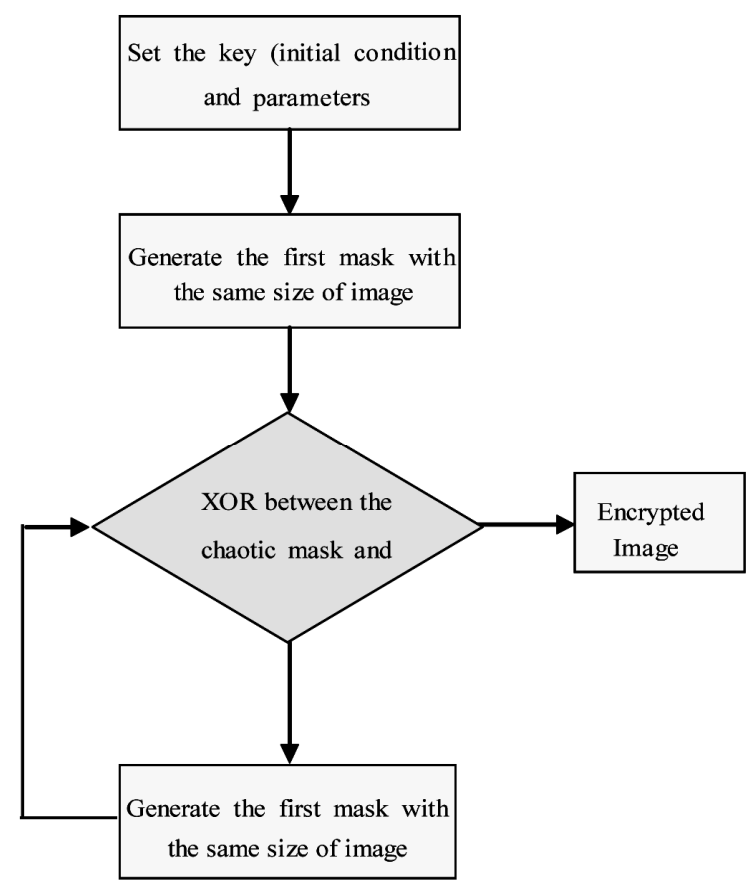

Figure 4. Flow chart of encryption based multiple chaotic procedure. section. We take $256 \times 256$ size 8 bits Lena image an example Figure 5(a) its encrypted image with the encryption initial parameters are $\left(\delta, \beta, r, a, b, c, y_{0}, x_{0}, z_{0}\right)$ $=(16,45.6,2,0.2,0.2,5.7)$ but about initial condition we divided initial condition for each generated mask $\left(x_{10}\right.$, $\left.x_{20}, x_{30}\right)=(0.832345676543451,0.523456765475432$, 0.6345677654345643 ) as we can see, the encrypted image is rough-and-tumble, unknowable and $100 \%$ obscure of the image Figure 5(b) is the decrypted image by use the same encryption key. It can be seen that the decrypted image is clear and correct without any distortion see Figure 5(c) to see no error between original image and decrypted image.

In Figure 6 their encrypted image with sharp edge with the same encryption initial parameters and initial conditions. As we can see, the encrypted image is rough-andtumble, unknowable and $100 \%$ obscure of the image Figure 6(c) is the decrypted image by use the same encryption key. It can be seen that the decrypted image is clear and correct without any distortion see Figure 6(c) to see no error between original image and decrypted image.

\section{Statistical Analysis}

Use Statistical analysis has been performed on the proposed image encryption algorithm, demonstrating its superior confusion and diffusion properties which strongly resist statistical attacks. This is shown by a test on the histograms of the unencrypted images and on the correlations of adjacent pixels in the encrypted image.

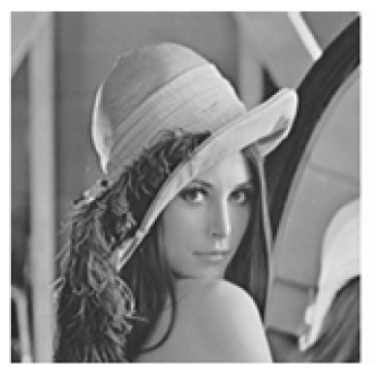

(a)

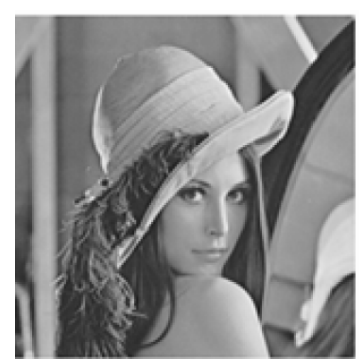

(c)

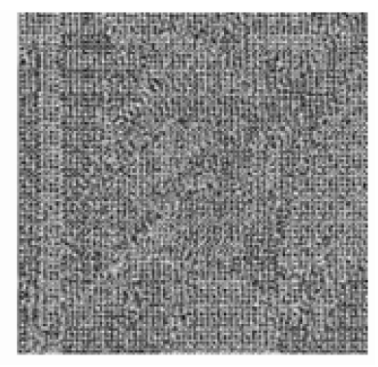

(b)

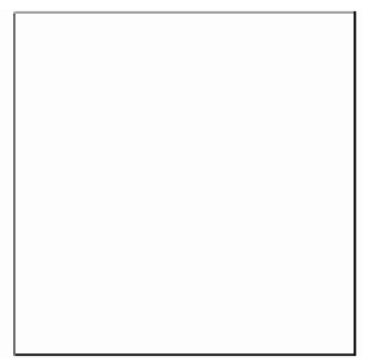

(d)
Figure 5. (a) Original image (b) encrypted image, (c) decrypted image (d) error between original and encrypted image. 


\section{Image}

(a)

\section{Image}

(c)

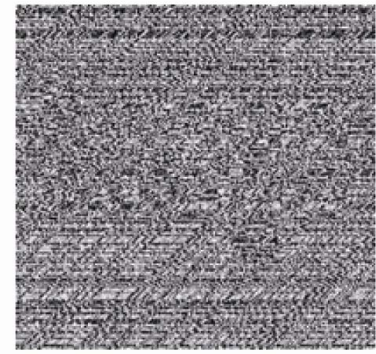

(b)

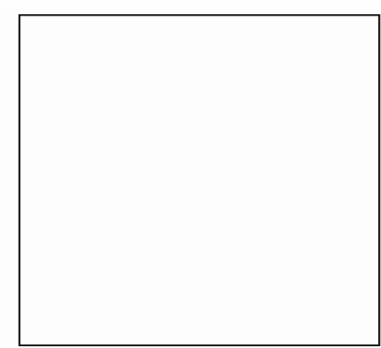

(d)

Figure 6. (a) Original Image (b) encrypted image, (c) decrypted image (d) error between original and encrypted image.

Histograms of encrypted images: one typical example among them is shown in Figures 7 and 8; one can see that the histogram of the encrypted image for Lena image is fairly uniform and is significantly different from that of the original image.

We have also done extensive study of the correlation between image and its corresponding encrypted image by using the proposed encryption algorithm. The correlation between two vertically adjacent pixels, two horizontally adjacent pixels, and two diagonally adjacent pixels, We have depicted the distributions of two horizontally, vertically and diagonally adjacent pixels in the original and encrypted images respectively see Figure $\mathbf{9}$ and Table $\mathbf{1}$ for Lena image and Figure $\mathbf{1 0}$ and Table $\mathbf{2}$ for sharp edge image, we note the two adjacent pixels in the original image are highly correlated, and the two adjacent pixels in the encrypted image are highly not correlated.

$$
E(x)=\frac{1}{N} \sum_{i=1}^{N} x_{i}
$$

\section{Differential and Sensitivity Analysis}

Another important test is measured the number of pixels change rate (NPCR) to see the influence of changing a single pixel in the original image on the encrypted image by the proposed algorithm defined by Equation (7). The NPCR measure the percentage of different pixel numbers between the two images and the UACI (unified average changing intensity) defined by Equation (8). We take two
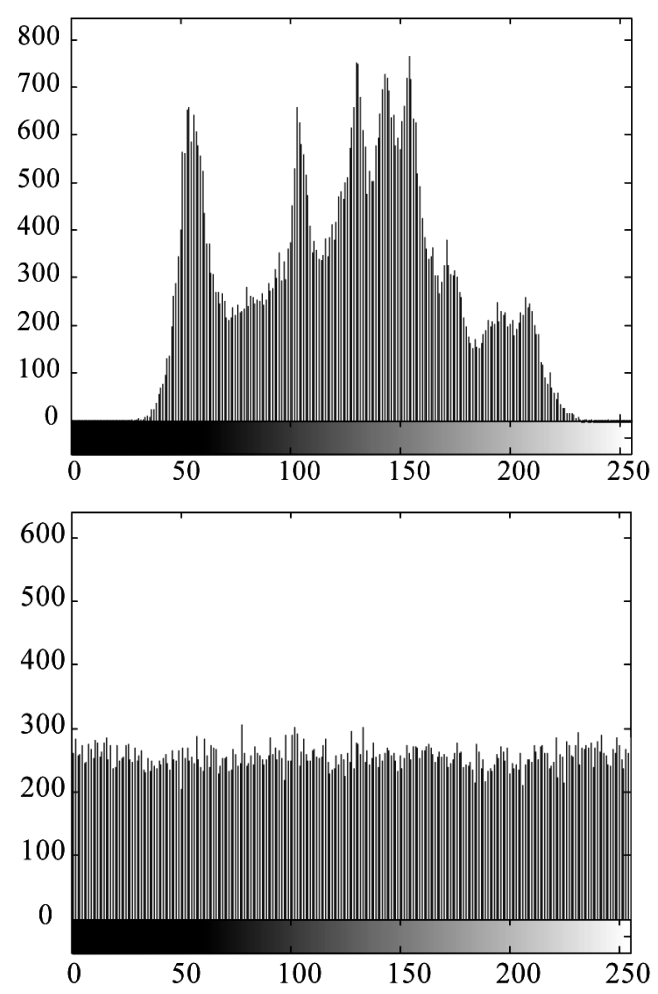

Figure 7. Histogram of original image and encrypted for lena image.
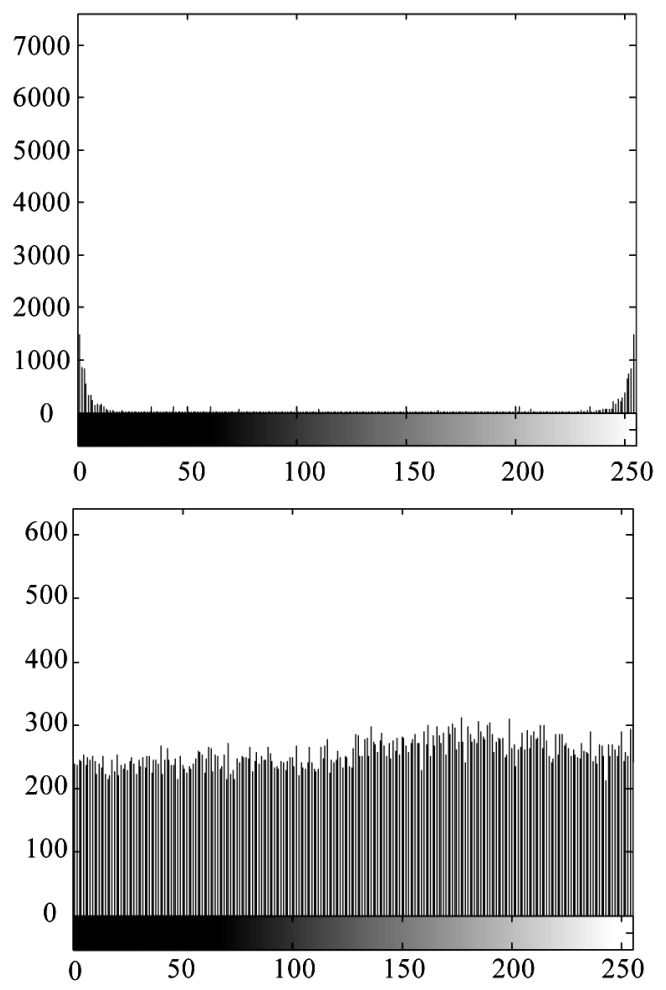

Figure 8. Histogram of original image and encrypted for sharp edge image. 

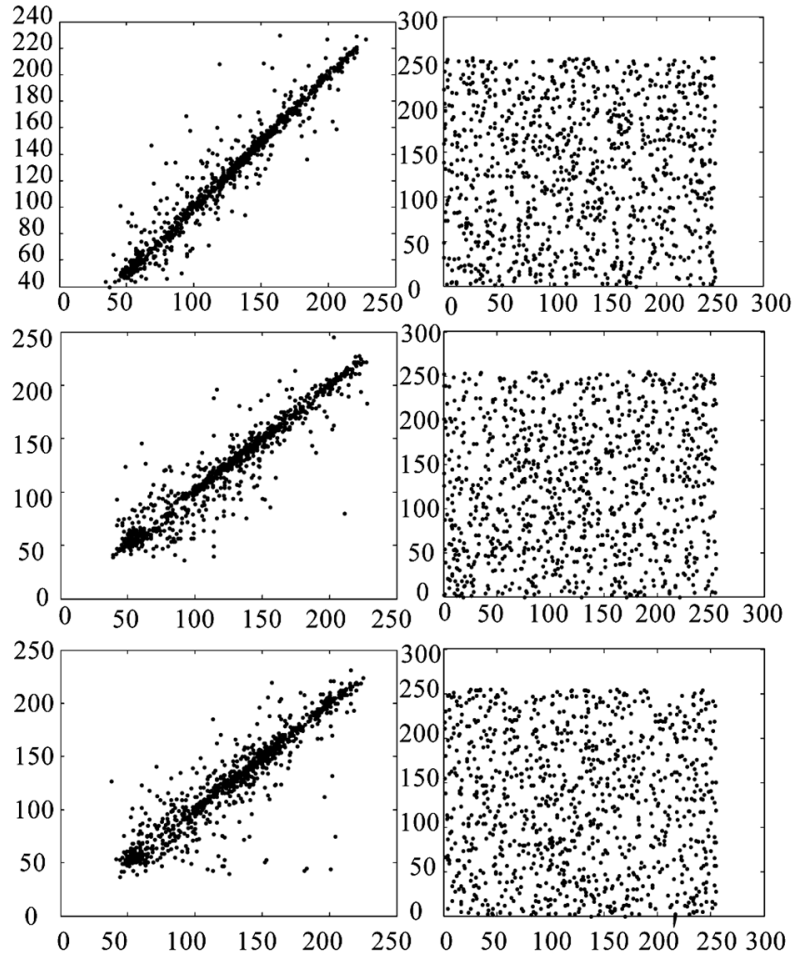

Figure 9. Correlations of two diagonal, horizontally and vertical adjacent pixels in the plain image and in the encrypted-image.

Table 1. Correlations coefficient of two diagonal, horizontally and vertical adjacent pixels in the plain image and the encrypted-image.

\begin{tabular}{ccc}
\hline & Plain image & Encrypted image \\
\hline Horizontal & 0.9681 & 0.0483 \\
Vertical & 0.9434 & 0.1078 \\
Diagonal & 0.9238 & -0.0283 \\
\hline
\end{tabular}

encrypted images, C1 and C2, whose corresponding original images have only one-pixel difference. We define a two-dimensional array $\mathrm{D}$, having the same size as the image $\mathrm{C} 1$ and $\mathrm{C} 2$. If $C_{1}(i, j)=C_{1}(i, j)$ then $D(i, j)=1$, otherwise $D(i, j)=0$

$$
\begin{gathered}
N P C R=\frac{\sum_{i, j} D(i, j)}{W \times H} \times 100 \% \\
U A C I=\frac{1}{W \times H}\left[\sum_{i, j} \frac{\left|C_{1}(i, j)-C_{2}(i, j)\right|}{255}\right] \times 100 \%
\end{gathered}
$$

where $W$ and $H$ are the width and height of encrypted image. We obtained NPCR for a large number of images by using our encryption scheme and found it to be over $99 \%$ showing thereby that the encryption scheme is very
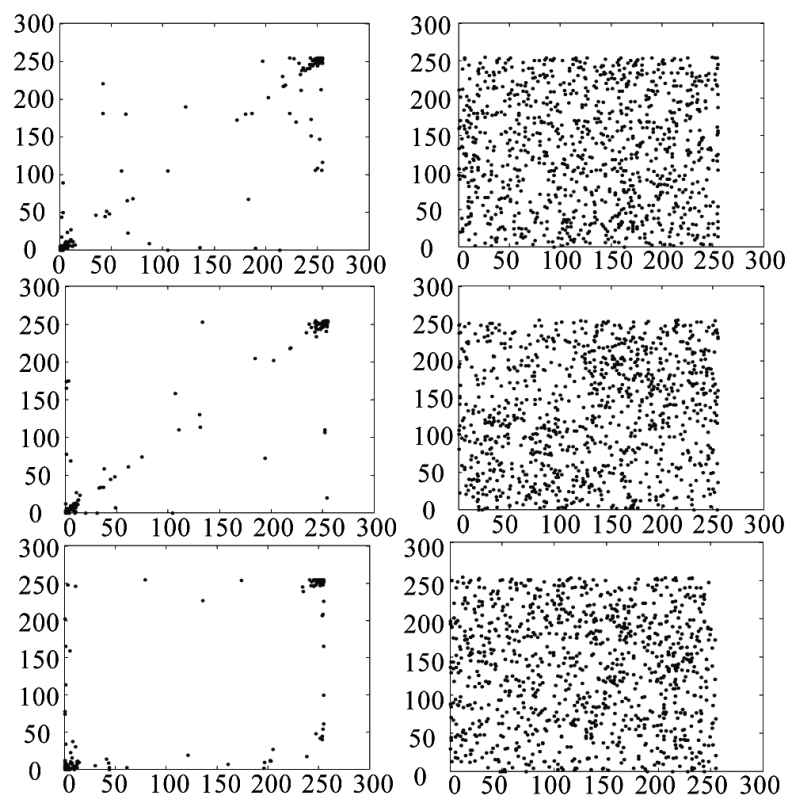

Figure 10. Correlations of two diagonal, horizontally and vertical adjacent pixels in the plain image and in the encrypted-image.

Table 2. Correlations coefficient of two diagonal, horizontally and vertical adjacent pixels in the plain image and the encrypted-image.

\begin{tabular}{ccc}
\hline & Plain image & Encrypted image \\
\hline Horizontal & 0.9804 & 0.0219 \\
Vertical & 0.9434 & 0.1668 \\
Diagonal & 0.9351 & -0.0024 \\
\hline
\end{tabular}

sensitive with respect to small changes in the plaintext $[14,15]$.

\section{Key Space Analysis}

Another secret key should produce a completely different encrypted image. For testing the key sensitivity of the proposed image encryption procedure, we use the wrong key, initial parameters to decrypted the original image for example if we encrypt the Lena image using $(\delta, \beta$, $\left.r, a, b, c, y_{0}, x_{0}, z_{0}\right)=(16,45.6,2,0.2,0.2,5.7)$ and $\left(x_{10}, x_{20}, x_{30}\right)=(0.832345676543451,0.523456765475432$, $0.6345677654345643)$ now we will try to decrypt the encrypted image using wrong key for example $(\delta, \beta, r$, $\left.a, b, c, y_{0}, x_{0}, z_{0}\right)=(16,45.6,2,0.2,0.2,5.7)$ and $\left(x_{10}\right.$, $\left.x_{20}, x_{30}\right)=(0.832345676543451,0.523456765475432$, $0.6345677654345642)$ we note the decrypted image still rough-and-tumble and unknowable see Figure 11(a) and if we chose another key initial parameters are $(\delta, \beta, r$, $\left(\delta, \beta, r, a, b, c, y_{0}, x_{0}, z_{0}\right)=(16,45.6,2,0.2,0.2,5.7)$ and $\left(x_{10}, x_{20}, x_{30}\right)=(0.832345676543451,0.523456765$ 
Table 3. Comparison between proposed method and recent methods.

\begin{tabular}{cccccccc}
\hline & Lorenz [8] & Rossler [9] & Logistic Map [10] & New Logistic Map [7] & The 3D cat map [11] & One D based [8] $\begin{array}{c}\text { Encryption Based } \\
\text { Multiple chaotic }\end{array}$ \\
\hline Key Space & $2^{158}$ & $10^{16}$ & $1.2 \times 10^{24}$ & $10^{45}$ & $2^{36}$ & $22^{53}$ & $10^{75}$ \\
Time & $10.84 \mathrm{~s}$ & & 0.33 & $0.5 \mathrm{~s}$ & $0.4 \mathrm{~s}$ & $12.27 \mathrm{~s}$ & $2 \mathrm{~s}$ \\
Obscure & $100 \%$ & $100 \%$ & $<100 \%$ & $<100 \%$ & $100 \%$ & $100 \%$ & $100 \%$ \\
\hline
\end{tabular}

4754329, 0.6345677654345643) the result is tumble and unknowable as shown in Figure 11(b).

For a secure image encrypted, the key space should be large enough to make the brute force attack infeasible [16]. The key of the new algorithm consists of three floating-point numbers. We note if we encrypt using 15 digits and just change the last digit the decrypted still unknown that mean we use the first 15 digits of a floating-point number, then there are $15+15+15=75$ uncertain digits. So the possible key number is $10^{45}$. Moreover the parameters $(\delta, \beta, r, a, b, c)$ are also used as the secret key. An image encrypted with such a long key space is sufficient for reliable practical use.

\section{Time Analysis}

Apart from the security consideration, running speed of the algorithm is also an important aspect for a good encryption algorithm. We have measured the encryption/ decryption rate of on 256 grey-scale images of size $256 \times$ 256 by using the proposed image encryption scheme. The time analysis has been done on Pentium-4 with 512 MB RAM computer. The average encryption/decryption time
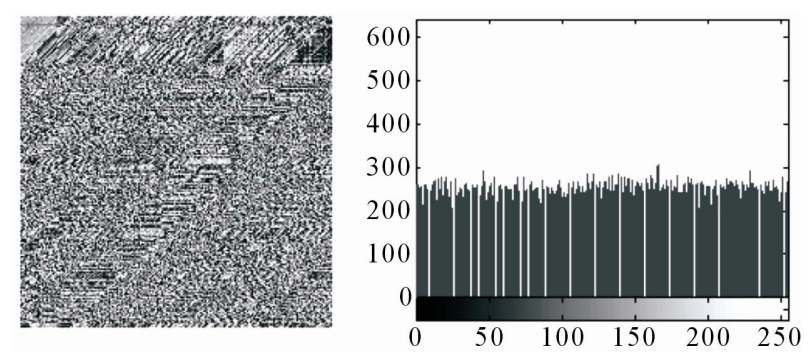

(a)
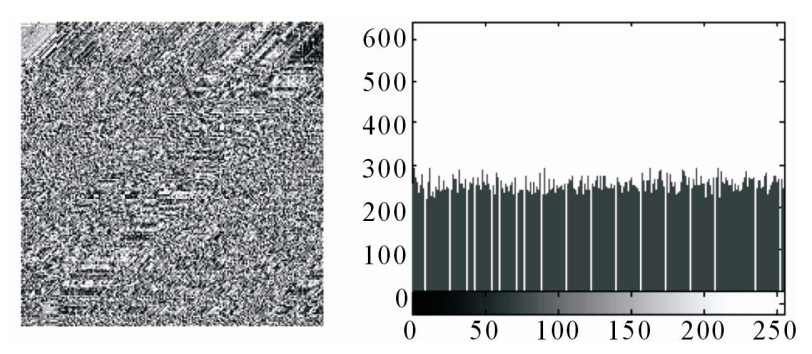

(b)

Figure 11. Decrypted using wrong key for lena image. is $2 \mathrm{~s}$ which is less than those of the previous algorithms. Comparison between proposed method and the previous methods as shown in Table 3.

\section{Conclusions}

This paper presents a new nonlinear chaotic algorithm, anew way of image encryption scheme have been proposed which uses two chaotic systems. To overcome the drawbacks of small key space and weak obscure in the current chaotic encryption methods, its structural parameters and initial value are used as encryption key in chaotic. Experimental analysis demonstrates that the image encryption algorithm has the advantages of large key space and high-level security, high obscure level and high speed. Finally, experimental and analytic results show that our scheme is efficient.

\section{REFERENCES}

[1] Z. Guan, F. Huang and W. Guan, "Chaos-Based Image Encryption Algorithm,” Physics Letters A, Vol. 346, No. 1-3, 2005, pp. 153-157. doi:10.1016/j.physleta.2005.08.006

[2] H. H. Nien, C. K. Huang, S. K. Changchien, H. W. Shieh, C. T. Chen and Y. Y. Tuan, "Digital Color Image Encoding and Decoding Using a Novel Chaotic Random Generator,” Chaos Solitons and Fractals, Vol. 32, No. 3, 2005, pp. 1070-1080. doi:10.1016/j.chaos.2005.11.057

[3] Q. Alsafasfeh and A. Alshabatat, "Image Encryption Based on Synchronized Communication Chaotic Circuit," Journal of Applied Sciences Research, Vol. 7, No. 4, 2011, pp. 392-399.

[4] H. Gao, Y. Zhang, S. Liang and D. Li, "A New Chaotic Algorithm for Image Encryption," Chaos Solitons and Fractals, Vol. 29, No. 2, 2006, pp. 393-399. doi:10.1016/j.chaos.2005.08.110

[5] C. Fu, Z. Zhang and Y. Cao, "An Improved Image Encryption Algorithm Based on Chaotic Maps,” Third International Conference on Natural Computation, Vol. 3, Washington, 2007, pp. 24-27.

[6] L. Zhang, X. Liao and X. Wang, “An Image Encryption Approach Based on Chaotic Maps,” Chaos Solitons and Fractals, Vol. 24, No. 3, 2005, pp. 759-765. doi:10.1016/j.chaos.2004.09.035

[7] S. Bu and B. Wang, "Improving the Security of Chaotic Encryption by Using a Simple Modulating Method," 
Chaos Solitons and Fractals, Vol. 19, No. 4, 2003, pp. 919-924. doi:10.1016/S0960-0779(03)00260-1

[8] X. Wu, H. Hu and B. Zhang, "Analyzing and Improving a Chaotic Encryption Method,” Chaos Solitons and Fractals, Vol. 22, No. 2, 2004, pp. 367-373. doi:10.1016/j.chaos.2004.02.009

[9] G. Chen, Y. Mao and C. K. Chui, "A Symmetric Image Encryption Scheme Based on 3D Chaotic Cat Maps," Chaos Solitons and Fractals, Vol. 21, No. 3, 2003, pp. 749-761. doi:10.1016/j.chaos.2003.12.022

[10] F. Chiaraluce, L. Ciccarelli, E. Gambi, P. Pierleoni and M. Reginelli, "A New Chaotic Algorithm for Video Encryption,” IEEE Transactions on Consumer Electronics, Vol. 48, No. 4, 2002, pp. 838-844. doi:10.1109/TCE.2003.1196410

[11] K. Wong, B. Kwok and W. Law, "A Fast Image Encryption Scheme Based on Chaotic Standard Map,” City University of Hong Kong, Hong Kong.

[12] L. Wang, Q. Ye, Y. Xiao, Y. Zou and B. Zhang, "An Image Encryption Scheme Based on Cross Chaotic Map,”
Congress on Image and Signal Processing, Sanya, 27-30 May 2008, pp. 27-26.

[13] Q. Alsafasfeh and M. Alarni, "A New Chaotic Attractor from Lorenz and Rossler Systems and Its Electronic Experimental Implementation,” Circuits and Systems, Vol. 2, No. 2, 2011, pp. 101-105. doi:10.4236/cs.2011.22015

[14] T. Ga and Z. Chen, "Image Encryption Based on a New Total Shuffling Algorithm,” Chaos Solitons \& Fractals, Vol. 38, No. 1, 2008, pp. 213-220. doi:10.1016/j.chaos.2006.11.009

[15] N. K. Pareek, V. Patidar and K. K. Sud, "Image Encryption Using Chaotic Logistic Map,” Image and Vision Computing, Vol. 24, No. 9, 2006, pp. 926-934. doi:10.1016/j.imavis.2006.02.021

[16] V. Patidar, N. K. Pareek and K. K. Sud, “A New Substitution-Diffusion Based Image Encrypete Using Chaotic Standard and Logistic Maps," Communications in NonLinear Science and Numerical Simulation, Vol. 14, No. 7, 2009, pp. 3056-3075. doi:10.1016/j.cnsns.2008.11.005 